\title{
TABELAS DE FERTILIDADE E DE ESPERANCCA DE VIDA DE TYNACANTHA MARGINATA DALLAS (HETEROPTERA, PENTATOMIDAE, ASOPINAE) ALIMENTADO COM LARVAS DE TENEBRIO MOLITOR L. (COLEOPTERA, TENEBRIONIDAE) E FOLHAS DE EUCALYPTUS UROPHYLLA S.T. BLAKE
}

\author{
Luciano Andrade Moreira ${ }^{1}$ \\ José Cola Zanuncio ${ }^{2}$ \\ Marcelo Coutinho Picanço ${ }^{2}$ \\ Claudio Horst Bruckner ${ }^{3}$
}

\begin{abstract}
LIFE AND FECUNDTTY TABLES OF THE PREDATOR TYNACANTHA MLAR CIINATA

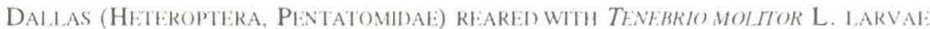
(COLEOPTERA, TENEBRIONIDAE) AND EUKALYPTIS IROPHylLA S.T. BLAKE LEAVES. The objective of this research was to study the effect of feeding on Eucalyptus leaves on the life and fecundity tables of Tynacantha marginata Dallas, 1851 (Heteroptera: Pentatonidae). Higher mortality of this predator occurred during second week of life, when the nymphs were starting second instar. The fecundity table showed that the nymphal period of $T$. marginata lasted four weeks, with viability of $57.9 \%$ and total longevity of 21 weeks. Egg oviposition period took 10 weeks. The population parameters $\left(\mathrm{R}_{(),} \mathrm{rm}\right.$ and $\lambda$ ) showed a 50.69 times populational increase after one generation.

KEY WORDS. Hetereptera, Tynacantha marginata. life tahles, predator
\end{abstract}

Tynacantha marginata Dallas, 1851 (Heteroptera, Pentatomidae, Asopinae) apresenta possibilidades de utilização em programas de controle biológico, tendo sido encontrado predando insetos pragas em vários estados brasileiros (BUCKUP 1960; MENDES 1959; ZANUNCIO et al. 1994). Alguns aspectos relacionados com a biologia desse predador foram estudados por TELLA (1951), ZACHRISSON (1987) e TEIXEIRA et al. (1989), incluindo o sell comportamento de sugar folhas de soja perene (GARCIA 1991).

Pela necessidade de estudo de maior número de espécies com possibilidade de utilização em nível de campo, visando o controle biológico de pragas em sistemas agrícolas e florestais, procurou-se estudar, em laboratório, as tahelas de vida de T. marginata, alimentado com larvas de Tenebrio molitor L., 1758 (Coleoptera, Tenehrionidae) e folhas de Eucalyptus urophylla S. T. Blake.

1) Secretaria de Genétiea e Melhoramento, Universidade Federal de Viçosa. 36571-000) Viçosá. Minas Gerais. Brasil.

2) Departamento de Biologia Animal. Universidade Federal de Viçosa. 36571-000 Viçosa. Minas Gerais, Brasil.

3) Departamento de Fitotecnia. Universidade Federal de Viçosa. 36571-000 Viçosa. Minas Gerais. Brasil. 


\section{MATERIAL E MÉTODOS}

O experimento foi conduzido no Laboratório de Entomologia Florestal, do Departamento de Biologia Animal, Universidade Federal de Viçosa, Minas Gerais.

$\mathrm{Na}$ segunda geração do predador, o experimento foi montado, utilizando-se uma incubadora BOD, à $25 \pm 0,5^{\circ} \mathrm{C}$, fotoperíodo $12 \mathrm{~L}$ : $12 \mathrm{E}$ e $65 \pm 10 \%$ UR. Foram retirados, ao acaso, 25 ovos, com coloração amarelo ouro, pois provavelmente estariam férteis (TeIXEIRA et al. 1989) de cada uma das seis posturas de $T$. marginata, disponíveis na data do início do experimento. As ninfas de primeiro estádio, receberam folhas de $E$. urophylla e água destilada.

No segundo estádio, as ninfas foram individualizadas e colocadas em copos plásticos de $200 \mathrm{ml}$, fechados com tampa cuja parte central foi substituída por tela de náilon de $1 \mathrm{~mm}$ de malha (ZANUNCiO et al. 1992). O fornecimento de água foi realizado inserindo-se um tubo de anestésico (dentista) com a boca voltada para dentro e tampada com um chumaço de algodão, onde inseriu-se o pecíolo de uma folha de E. urophylla. Foi mantida, dentro dos copos de criação, uma larva de último estádio de $T$. molitor, sendo as folhas de eucalipto trocadas a cada dois dias. Realizaram-se observações diárias, anotando-se as mudanças de estádio e a mortalidade dos insetos. Para cálculo das tabelas de vida utilizaram-se os dados semanais.

A elaboração das tabelas de vida foi feita segundo SouTHWood (1978). Determinou-se a esperança de vida para metade da população (ex 50 ), por análise de próbite (FINNEY 1971).

A análise de sobrevivência foi realizada segundo Southwond (1978) e a confecção da tabela de fertilidade, de acordo com RaBINOVICH (1978).

\section{RESULTADOS E DISCUSSÃO}

A viahilidade de ovos de T. marginata foi de $100 \%$ e da fase ninfal de $55,49 \%$. Como a segunda semana mostra $100 \%$ de sobreviventes e Ix se relaciona ao valor médio de sobreviventes no início de cada idade $x$, a viabilidade de ninfas consistiu na divisão de lx da sexta pela segunda semana de vida (Tah. I). Esse valor foi semelhante ao obtido por ZanUnCIO et al. (1993) para Podisus nigrispinus (=Podisus connexivus Bergroth, 1891) (Heteroptera: Pentatomidae). A longevidade máxima (de ovo até a morte dos adultos) de T. marginata foi de 21 semanas.

A esperança de vida foi de 9,024 e 8,024 semanas para os ovos e ninfas de primeiro estádio, respectivamente; enquanto os adultos recém-emergìdos, apresentaram para esta característica, 9,887 semanas (Tab. I), que reflete a expectativa de vida em cada intervalo de idade (RABINOVICH 1978).

Observou-se decréscimo da esperança de vida ao longo do ciclo do inseto (Fig. 1), mas apesar desta tendência, determinados intervalos de idade apresentaram menores valores de esperança de vida que intervalos posteriores. Isto ocorreu para ninfas recém eclodidas e com adultos de 10 e 13 semanas de idade, representando períodos críticos de riscos de mortalidade para determinada população (RABINOVICH 1978). 
Tabela I. Tabela de esperança de vida para Tynacantha marginata (Heteroptera, Pentatomidae), alimentado com larvas de Tenebrio molitor (Coleoptera, Tenebrionidae) e folhas de Eucalyptus urophylla, em laboratório, $\mathrm{T}=25 \pm 0,5^{\circ} \mathrm{C}$, fotoperiodo $12 \mathrm{~L}: 12 \mathrm{E}$ e $65 \pm 10 \%$ UR. Viçosa, Minas Gerais, 1992.

\begin{tabular}{|c|c|c|c|c|c|c|c|}
\hline$x$ & Ix & $d x$ & $L x$ & $T x$ & ex & $100 q x$ & Estágio \\
\hline 1 & 100,000 & 0,000 & 100,000 & 902,490 & 9,024 & 0,000 & ovo \\
\hline 2 & 100,000 & 39,690 & 80,155 & 802,490 & 8,024 & 39,690 & \\
\hline 3 & 60,310 & 2,410 & 59,105 & 722,335 & 11,977 & 3,996 & $\operatorname{ninfa}$ \\
\hline 4 & 57,900 & 0,000 & 57,900 & 663,230 & 11,454 & 0,000 & \\
\hline 5 & 57,900 & 2,410 & 56,695 & 605,330 & 10,454 & 4,162 & \\
\hline 6 & 55,490 & 2,410 & 54,285 & 548,635 & 9,887 & 4,343 & \\
\hline 7 & 53,080 & 0,000 & 53,080 & 494,350 & 9,313 & 0,000 & \\
\hline 8 & 53,080 & 0,000 & 53,080 & 441,270 & 8,313 & 0,000 & \\
\hline 9 & 53,080 & 0,000 & 53,080 & 388,190 & 7,313 & 0,000 & \\
\hline 10 & 53,080 & 6,630 & 49,765 & 335,110 & 6,313 & 12,491 & \\
\hline 11 & 46,450 & 3,320 & 44,790 & 285,345 & 6,143 & 7,147 & \\
\hline 12 & 43,130 & 0,000 & 43,130 & 240,555 & 5,577 & 0,000 & \\
\hline 13 & 43,130 & 3,320 & 41,470 & 197,425 & 4,577 & 7,698 & adulto \\
\hline 14 & 39,810 & 0,000 & 39,810 & 155,955 & 3,917 & 0,000 & \\
\hline 15 & 39,810 & 13,270 & 33,175 & 116,145 & 2,917 & 33,333 & \\
\hline 16 & 26,540 & 6,630 & 23,225 & 82,970 & 3,126 & 24,981 & \\
\hline 17 & 19,910 & 0,000 & 19,910 & 59,745 & 3,000 & 0,000 & \\
\hline 18 & 19,910 & 9,950 & 14,935 & 39,835 & 2,000 & 49,975 & \\
\hline 19 & 9,960 & 0,000 & 9,960 & 24,900 & 2,500 & 0,000 & \\
\hline 20 & 9,960 & 0,000 & 9,960 & 14,940 & 1,500 & 0,000 & \\
\hline 21 & 9,960 & 9,960 & 4,980 & 4,980 & 0,500 & 100,000 & \\
\hline
\end{tabular}

$\mathbf{x}=$ Idade (semanas); $\mid \mathbf{x}=$ número de insetos sobreviventes no início da idade; $\mathbf{d} \mathbf{x}=$ número de insetos mortos entre as idades $x$ e $x+1 ; L x=$ número de insetos vivos entre $\mathrm{x}$ e $\mathrm{x}+1 ; \mathbf{T} \mathbf{x}=$ número total de semanas que restam para viver aos sobreviventes que têm alcançado a idade $\mathrm{x}$; $\mathrm{ex}=$ esperança de vida; $100 \mathrm{qx}=$ Probabilidade de morrer entre $x$ e $x+1$.

Ohteve-se 7,81 semanas para a esperança de vida da metade da população (ex50) dos percevejos, por meio de análise de próbite, sugerindo que deve ser dado maior empenho no início da fase adulta dos percevejos, para que haja maior produção de ovos.

A sobrevivência de $T$. marginata mostra uma curva mesclada dos tipos I e IV, conforme SouThwood (1978) (Fig. 2), semelhante a do percevejo hematófago Triatoma infestans (Klug., 1834) (Heteroptera, Reduviidae), que apresenta queda brusca nas fases iniciais de vida, correspondendo à eclosão dos ovos e a mudança do primeiro estádio das ninfas (RABINOviCH 1978). No final do experimento, a taxa de mortalidade se intensificou provavelmente devido à elevada idade dos adultos.

A tabela de vida de fertilidade de $T$. marginata (Tah. II) mostra que a oviposição se iniciou na sétima e se estendeu por 10 semanas. Obteve-se um total 


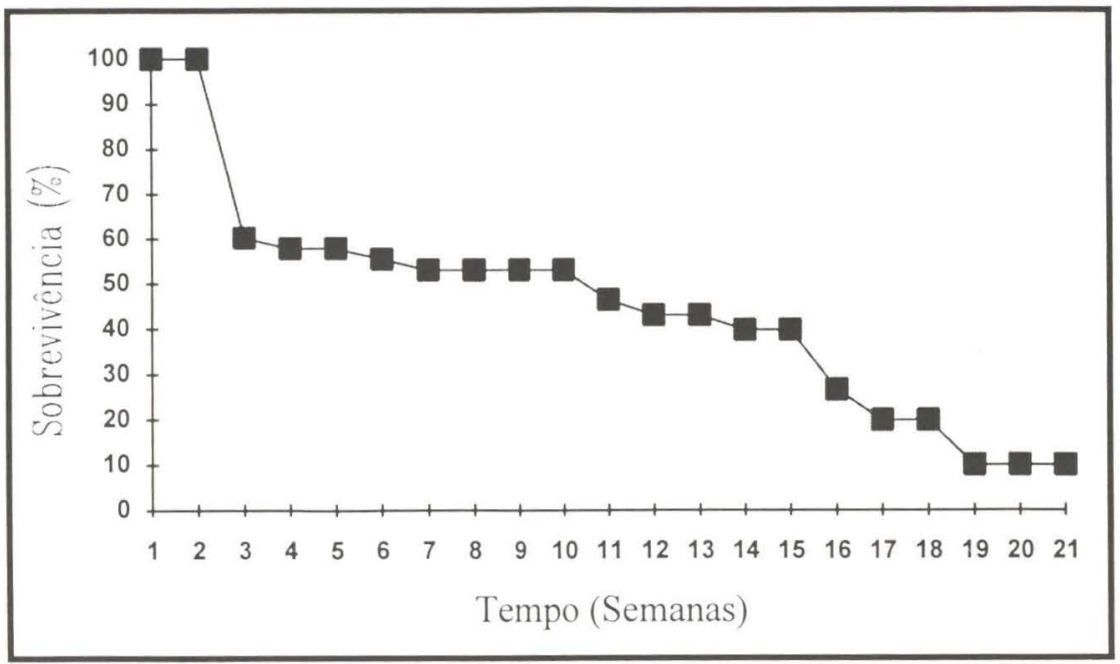

Fig. 1. Curva de esperança de vida de Tynacantha marginata alimentado com larvas de Tenebrio molitor e folhas de Eucalyptus urophylla.

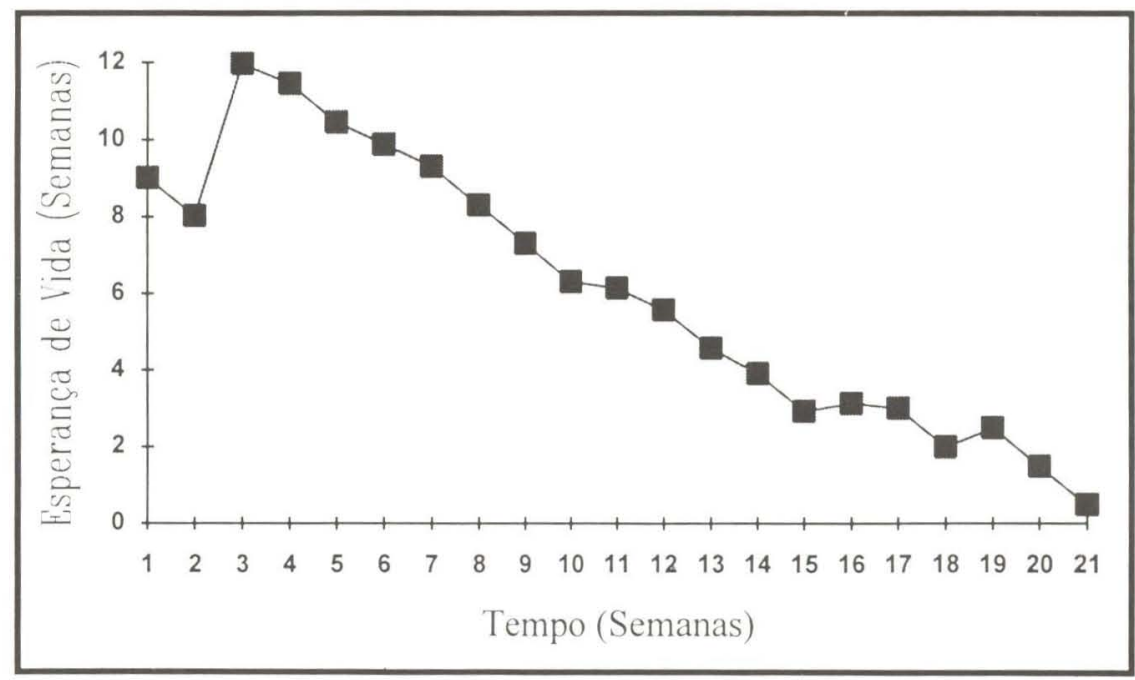

Fig. 2. Curva de sobrevivência de Tynacantha marginata alimentado com larvas de Tenebrio molitor e folhas de Eucalyptus urophylla.

de 1527 ovos (considerando-se razão sexual 1:1 e oito fêmeas em reprodução) e, média de 190,87 ovos por fêmea. A produção de ovos foi crescente da sétima à décima semana, mantendo-se mais baixa até a $16^{\mathrm{a}}$ semana, o que é interessante no caso de utilização desse predador em programas de controle biológico. Picos de oviposição podem não ser favoráveis, quando não coincidem com a disponibilidade de pragas, no campo. 
Tabela II. Tabela de vida de fertilidade de Tynacantha marginata (Heteroptera, Pentatomidae), alimentado com larvas de Tenebrio molitor (Coleoptera, Tenebrionidae) e folhas de Eucalyptus urophylla, em laboratório, $\mathrm{T}=25 \cdot 0,5^{\circ} \mathrm{C}$, fotoperíodo $12 \mathrm{~L}: 12 \mathrm{E}$ e $65: 10 \%$ UR. Viçosa, Minas Gerais, 1992.

\begin{tabular}{|c|c|c|c|c|c|}
\hline$x$ & $m x$ & Ix & $m x \cdot I x$ & $m x \cdot 1 x \cdot x$ & Estágio \\
\hline 0,5 & 0,000 & 1,000 & 0,000 & 0,000 & ovo \\
\hline 1,5 & 0,000 & 0,802 & 0,000 & 0,000 & \\
\hline 2,5 & 0,000 & 0,591 & 0,000 & 0,000 & ninfa \\
\hline 3,5 & 0,000 & 0,579 & 0,000 & 0,000 & \\
\hline 4,5 & 0,000 & 0,567 & 0,000 & 0,000 & \\
\hline 5,5 & 0,000 & 0,543 & 0,000 & 0,000 & \\
\hline 6,5 & 9,190 & 0,531 & 4,880 & 31,720 & \\
\hline 7,5 & 12,310 & 0,531 & 6,537 & 49,027 & \\
\hline 8,5 & 13,380 & 0,531 & 7,105 & 60,392 & \\
\hline 9,5 & 24,870 & 0,498 & 12,385 & 117,657 & \\
\hline 10,5 & 6,140 & 0,448 & 2,751 & 28,885 & \\
\hline 11,5 & 21,000 & 0,431 & 9,051 & 104,086 & \\
\hline 12,5 & 4,770 & 0,415 & 1,978 & 24,725 & adulto \\
\hline 13,5 & 11,580 & 0,398 & 4,608 & 62,208 & \\
\hline 14,5 & 0,000 & 0,332 & 0,000 & 0,000 & \\
\hline 15,5 & 6,000 & 0,232 & 1,392 & 21,576 & \\
\hline 16,5 & 0,000 & 0,199 & 0,000 & 0,000 & \\
\hline 17,5 & 0,000 & 0,149 & 0,000 & 0,000 & \\
\hline 18,5 & 0,000 & 0,100 & 0,000 & 0,000 & \\
\hline 19,5 & 0,000 & 0,100 & 0,000 & 0,000 & \\
\hline 20,5 & 0,000 & 0,050 & 0,000 & 0,000 & \\
\hline
\end{tabular}

ะ $50,687 \quad 500,276$

$T=9,87$ semanas, $R_{0}=50,69$ filhas $/$ fêmea, $r m=0,3977 ; \lambda=1,4884$ ind. $/$ fêmea $/$ se mana.

$\mathbf{x}=$ Ponto médio da idade (semanas); $\mathbf{m x}$ = número total de ovos por fêmea, por semana, e que darão fêmeas; $\mid \mathbf{x}=$ proporção de fêmeas que vivem até a idade $\mathbf{x} ; \mathbf{R} \mathbf{0}=$ taxa líquida de reprodução; $\mathrm{T}=$ tempo médio de geração; $i=$ razão finita de aumento.

O tempo médio de geração, do nascimento de fêmeas ao nascimento de descendentes fêmeas (Southwood 1978) foi de 9,87 semanas, mostrando yue $T$. marginata pode ter eerca de cinco gerações por ano. Esse valor de $T$ é menor do que aqueles citados por RABINoVICH (1978), para Reduviidae (aproximadamente 200 dias) e Lygaeidae (maior que 100 dias), valores estes maiores que 14 semanas.

A taxa líquida de reprodução $\left(\mathrm{R}_{0}\right)$, de 50,69 filhas por fêmea (Tab. II), mostrou crescimento populacional, de $T$. marginata, de uma geração para outra, concordando com valores mostrados por RABINovicH (1978), eujos heterópteros possuíam $R_{0}$ sempre superiores a 1,0.

O potencial de crescimento populacional (rm) (SILVEIRA NeTo ét al. 1976) de $T$. marginata alimentado com larvas de $T$. molitor e folhas de $E$. arophylla foi de 0,3977 . Para a utilização em programas de controle biológico, é interessiante que um predador tenha aumentos populacionais, o que difere de casos em yue se 
trabalha com populações de pragas como grilos (Meloo et al. 1980) e com variedades de plantas resistentes à pragas, como para ácaros em algodão (TRICHILo \& LEIGH 1985).

O valor de i representa a razão finita de aumento e se diferencia da taxa instântanea de aumento (rm). Isto confirma o valor de $\mathrm{R}_{0}$, cujo valor mostrou aumento populacional de uma geração para outra. O valor de 1,4884 representa a agregação de mais de um indivíduo, de uma geração para outra, é interpretado como o número de indivíduos que se soma à população, por têmea e por unidade de tempo (RABINovich 1978).

AGRADECIMENTOS. Ao BIOAGRO. CNPy e FAPEMIG pelas bolsas e auxilios concedidos. Ao Programa Cooperativo de Mancjo Integrado de Pragas Florestais (PC-MIP) da Sociedade de Investigaçôes Florestais pelo apoio para a realização dessa pesquisa.

\section{REFERÊNCIAS BIBLIOGRÁFICAS}

Buckup, L. 1960. Pentatomídeos Neotropicais. II. Iheringia, Sér. Zool.(15): $1-25$.

Garcia, M.A. 1991. Ecologia Nutricional de Parasitóides e Predadores Terrestres. In: A.R. PANIzZI \& J.R.P. PARRA (ed.). Ecologia nutricional de insetos e suas implicações no manejo de pragas. São Paulo, Manole, 359 p.

Finney, D.J. 1971. Probit analysis. London, Cambridge University, 333p.

Mello, A.M.L.T.; S. Silveira Neto \& J.R.P. Parra. 1980. Tabela de fertilidade e esperança de vida de Gryllus assimilis (Fah., 1775) (Orthoptera: Gryllidae) em laboratório. An. Soc. Entomol. Brasil 9 (1): 133-140.

MENDES, L.O.T. 1959. Sobre a ocorrência de alģuns inimigos naturais de insetos.

An. Acad. Bras. Cien. 31 (4): 577-585.

Rabinovich, J.E. 1978. Ecologia de poblaciones animales. Caracas, Instituto Venezoelano de Investigationes Cientificas, 114p.

Silveira Neto, S.; O. Nakano; D. Barbin \& N.A. Villa Nova. 1976. Manual de ecologia dos insetos. São Paulo, Agronômica Ceres, 419p.

SouThWo()D, T.R.E. 1978. Ecological methods with particular reference to the study of insect populations. London, Chapman and Hall, 391p.

Teixeira. C.A.D.; W.G. Campos; J.C. Zanuncio \& V. Castro. 1989. Aspectos do ciclo evolutivo de Tynacantha marginata (Hemiptera: Pentatomidae) em dieta à base de Bombyx mori (Lepidoptera: Bombycidae). Resumos do XII Congresso Brasileiro de Entomologia, Belo Horizonte, p.447.

TELla, R. DE. 1951. Dados bionômicos de um percevejo predador. Bragantia 11 (4/6): 179-80.

Trichilo, P.J. \& T.F. Leigh. 1985. The use of life tables to access varietal resistance of cotton to spider mites. Entomol. Exp. Appl. 39: 27-33.

Zachrisson, B.A. 1987. Aspectos biológicos do predador Tynacamtha marginata Dallas, 1851 (Pentatomidae: Asopinae), sob condições de lahoratório. Resumos do XI Congresso Brasileiro de Entomologia, Campinas, p. 183. 
Zanuncio, J.C.; J.B. Alves; R.C. Sartório \& J.E.M. Leite. 1992. Métodos de criação de hemípteros predadores de lagartas. An. Soc. Entomol. Brasil 21 (2): $245-251$.

Zanunci(), J.C.; A.T. Ferreira; T.V. Zanunclo \& J.F. Garcia. 1993. Influence of feeding on Eucalyptus urophylla seedlings on the development of the predatory hug Podisus connexivus (Hemiptera: Pentatomidae). Med. Fac. Landhouww. Univ. Gent 58 (2a): 469-475.

Zanunci( ), J.C.; J.B. Alves; T.V. Zanuncio \& J.F. Garcia. 1994. Hemipterous predators of eucalypt defoliator caterpillars. Forest Ecology and Management 65 (1): 65-73. 